Conde-Sala, J. L. (2003). Factores de riesgo psicosocial y personalidad premórbida en enfermos con demencia, un estudio de casos y controles [Psychosocial risk factors and premorbid personality in patients with dementia: a case-control study]. Revista Española de Geriatría y Gerontología 2003; 38 (1): 10-24.

Factores de riesgo psicosocial y personalidad premórbida en enfermos con demencia: un estudio caso-control

Psychosocial risk factors and premorbid personality in patients with dementia:

a case-control study

Datos del autor:

J. Lluís Conde Sala

Doctor en Psicología

Universidad de Barcelona

\title{
Correspondencia:
}

Departamento de Psicología Evolutiva. Facultad de Psicología. Universidad de Barcelona

Paseo del Valle de Hebrón, 171 - 08035 BARCELONA -

Tf. 9331258 14; e-mail: jllconde@ub.edu 


\section{Factores de riesgo psicosocial y personalidad premórbida en enfermos con demencia: un estudio caso-control}

\section{RESUMEN}

Objetivos: Explorar los factores de riesgo no-biológicos para las demencias, plantear probables perfiles de riesgo para la enfermedad de Alzheimer y sugerir elementos para un posible desarrollo de intervenciones preventivas.

Metodología: Se administró un cuestionario sobre eventos vitales y factores de personalidad, elaborado específicamente, a un grupo de enfermos (108 personas con demencia) y un grupo control (49 personas mayores sin demencia), identificando las diferencias significativas con un nivel alfa de 0.05 para todas las pruebas estadísticas.

Resultados: Como probables factores de riesgo para la enfermedad de Alzheimer señalar: 1) Aspectos psíquicos: mayor número de trastornos psíquicos familiares graves y mayor respuesta psicosomática personal; 2) Aspectos familiares: mayor número de muertes precoces del padre; 3) Educación y trabajo: menor nivel educativo y menor cualificación en el trabajo; 4) Eventos vitales: mayor problemática familiar; 5) Factores de personalidad según el criterio de los cinco grandes: Energía (Imitación, evitación, dependencia general y de relación), Afabilidad (Introversión), Estabilidad emocional (Afectación y pesimismo), Apertura mental (Indiferencia y rigidez).

Conclusiones: Destacar la relevancia del porcentaje de muertes precoces del padre y el déficit de personalidad en el factor I (Energía): Imitación, evitación y dependencia. Como posibles medidas de prevención se sugieren intervenciones que potencien la autonomía personal, la capacidad de afrontamiento y la relación social.

Palabras clave: Alzheimer, Demencia, Factores de riesgo, Personalidad premórbida, Psicosocial 


\section{Psychosocial risk factors and premorbid personality in patients with dementia: a case- control study}

\section{SUMMARY:}

Objectives: To explore the non-biological risk factors for dementias; to suggest probable risk profiles for Alzheimer's disease; to propose elements for a possible development of preventive interventions.

Methodology: Empirical comparison of a dementia group (108 patients) and a control group (49 individuals without dementia). A specific questionnaire was administered and were identified the significant differences. An alpha level of 0.05 was used for all statistical tests.

Results: As probable risk factors for Alzheimer's disease to indicate: 1) Psychiatric aspects: Serious psychiatric disorders in family members and high psychosomatic response; 2) Family aspects: Early death of father; 3) Education and work: Low level of schooling and predominance of low-skilled workers; 4) Life events: Family problems; 5) Factors of personality according to criterion of the big five: Energy (Imitation, avoidance, general dependence and relation dependence), Agreeableness (Introversion), Emotional stability (Affectation and pessimism), Openness (Nonchalance and inflexibility).

General conclusions: The most relevant is the high incidence of early death of father and personality deficits in the factor I (Energy): imitation, avoidance, dependence. As possible preventive measures they are suggested to promote personal autonomy, the ability to cope, and relations with others.

Key words: Alzheimer's disease, Dementia, Risk factors, Premorbid personality, Psychosocial 


\section{Introducción}

\section{Antecedentes y objetivos generales}

La investigación presentada ${ }^{1}$ tiene como objeto de estudio los factores psicosociales y de personalidad que presentan un alto grado de correlación con las demencias.

La motivación del estudio se produjo en el contexto de las entrevistas realizadas a familiares cuidadores de enfermos de Alzheimer, con los cuales el autor desarrollaba una tarea de apoyo educativo y emocional ${ }^{2}$. Algunos familiares insistían en que algo de lo que le sucedía al enfermo en la actualidad había estado presente en ellos, mucho antes de que desarrollase la enfermedad. Se referían a un modo de comportarse, consubstancial a ellos mismos, y que parecía haber estado como latente en etapas anteriores; a veces se referían también a un determinado evento vital como factor desencadenante.

El propósito inicial era, por tanto, conocer si podía justificarse la existencia de factores de riesgo nobiológicos, tal como señalaban algunos familiares. La revisión de los estudios previos sobre el tema confirmó al autor en la tarea de analizar otros factores englobados en el término genérico de factores medioambientales ${ }^{3,4}$. El propósito último tendría relación con el posible desarrollo de técnicas de intervención y políticas de salud pública con la población de riesgo, cuyo objetivo sería la prevención de las demencias para los colectivos específicos.

\section{Investigaciones previas sobre factores de riesgo en demencias}

Los estudios sobre los factores de riesgo en demencia se han concentrado de forma mayoritaria en el ámbito biológico. Aquellos que merecen un mayor consenso son: la edad creciente, la historia familiar 
de demencia, la presencia del síndrome de Down en los antecedentes familiares y más recientemente el genotipo Apo-E4 5 .

Por otra parte, se han ido incrementando también los estudios sobre los factores psicosociales y de personalidad, aunque sin llegar a conclusiones aceptadas globalmente. En la revisión de los estudios realizados ${ }^{6}$, algunos señalaban determinados factores de riesgo psicosociales para la enfermedad de Alzheimer: bajo estatus socio-económico ${ }^{7}$, menor nivel educativo ${ }^{8,9}$, menor grado de actividad y relación social $^{10-14}$ y presencia de eventos vitales negativos previos al desarrollo de la enfermedad ${ }^{15-16}$. Otros señalaban específicamente rasgos de personalidad premórbidos. En la Universidad de Lund ${ }^{17}$, se constataba que respecto a los conceptos: Validez (autoconfianza - inseguridad) y Solidez (firmeza sugestibilidad), aquellas personas que se desviaban de la media, tenían más riesgo de padecer la enfermedad de Alzheimer. En la Universidad de Friburgo ${ }^{18}$, encontraban rasgos del tipo: tendencia a la evitación - sumisión, a depender y pedir permiso a otros en decisiones importantes y unas relaciones sociales restringidas. En la Clínica Mayo ${ }^{19}$, evidenciaban la presencia de rasgos de introversión y pesimismo en la personalidad premórbida. Desde la Universidad de Viena ${ }^{20}$, se señalaba que no tener un estilo de trabajo activo y la vida con un esposo/a dominante, serían factores de riesgo para la enfermedad de Alzheimer. Recientemente, el estudio de las "Monjas" ${ }^{21}$ llegaba a la conclusión de que las emociones positivas y el optimismo ante la vida eran factores que protegían del Alzheimer.

\section{Planteamiento, hipótesis y objetivos específicos}

El estudio contemplaba la investigación sobre la presencia de los factores de riesgo en un grupo de enfermos con demencia y el contraste con un grupo control formado por personas sin demencia. Señalar que un primer problema lo constituía la imposibilidad de obtener información de forma directa 
de los enfermos por lo cual tuvo que recurrirse a los familiares más próximos. Los estudios ${ }^{22-25}$, sobre la fiabilidad de la información de los familiares, consideraban que ésta era fiable y básicamente concordante con la ofrecida por otras personas, con un cierto sesgo para la temática de la depresión.

El planteamiento del problema partía de una hipótesis previa sobre la posibilidad de que aspectos psicosociales y de personalidad actuasen como factores de riesgo y fueran detectables antes del inicio de la enfermedad. La concepción de estos factores no sería de tipo etiológico, sino de predisposición. a la enfermedad. De una forma más específica la hipótesis contemplaba la posibilidad de encontrar déficits en el ámbito familiar y personal.

Los objetivos específicos fueron:

a) Contrastar los resultados de una muestra de personas con demencia con los obtenidos en otras investigaciones sobre diversos factores de riesgo: eventos vitales negativos, bajo nivel educativo, ocupación laboral poco cualificada, escaso grado de relación social, factores de personalidad: introversión, evitación, sumisión, pesimismo.

b) Detectar nuevos factores, especialmente en el ámbito de la historia familiar y personal: presencia de trastornos psíquicos y/o psicosomáticos familiares y personales, ambiente familiar problemático, rasgos dominantes en el carácter de los padres, muerte precoz de los padres y ampliación de los factores de personalidad premórbida de las personas con demencia: dependencia, falta de criterio propio, rigidez, poco interés hacia los temas externos a la familia, afectación, irritabilidad, etc.

c) Contribuir a la definición de los perfiles de riesgo psico - social para las demencias, especialmente para la enfermedad de Alzheimer.

c) Identificar elementos sobre los cuales pudieran desarrollarse posibles intervenciones de prevención. 


\section{Metodología}

El estudio puede concebirse como una investigación empírica, en parte de carácter clínico y en parte epidemiológico, basada en el contraste entre un grupo de enfermos y otro de control, a los cuales se les administró un mismo cuestionario sobre aspectos psicosociales y de personalidad, analizando estadísticamente las diferencias de los resultados.

\section{Participantes}

El grupo de enfermos estaba formado por 108 personas diagnosticadas de demencia por los servicios sanitarios especializados. El grupo de control estaba formado por 49 personas mayores sin demencia, valorados respecto a la ausencia de déficits cognitivos relevantes por el propio investigador. La procedencia y la tipología de los participantes figuran en la tabla 1.

TABLA 1. Distribución de los participantes: número, tipologías y procedencia

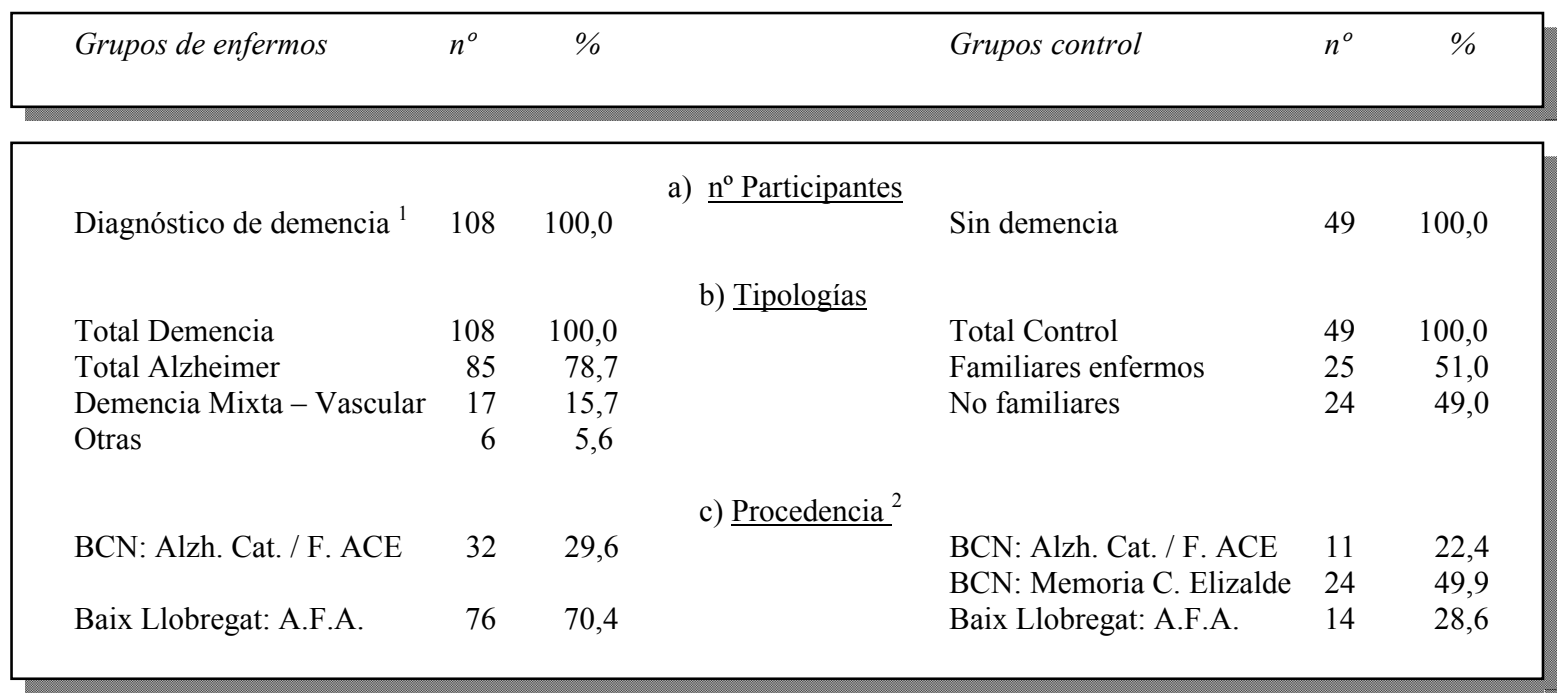

${ }^{1}$ Instituciones sanitarias de diagnóstico: Hospital de Bellvitge (28,7\%), Fundación ACE (25,9\%), CAP II de Cornellá (7,4\%), Hospital Clínico (4,6\%), Otros (33,4\%).

${ }^{2}$ BCN: Barcelona ciudad; Alzh. Cat. (Alzheimer Catalunya): Asociación de familiares y profesionales; AFA Baix Llobregat: Asociación de familiares de la comarca del Baix Llobregat; C. Elizalde: Centro Cívico, Ayuntamiento de Barcelona. 
En relación a la disimetría del tamaño del grupo de enfermos y el grupo control subrayar la dificultad para la captación aleatoria de los sujetos control, siendo por tanto, por razones clínicas, su acceso a ellos muy difícil y costoso. Señalar no obstante que el tamaño del grupo control es superior a 30, lo cual es considerado como una aproximación bastante buena a la distribución normal ${ }^{26-28}$. Por otra parte, constatar que en uno de los parámetros contrastados con el total de la población, la proporción de muertes precoces del padre antes de los 20 años de la edad del sujeto, la tasa del grupo control: $16,3 \%$, se comporta de forma parecida a la tasa estimada para la población normal española ${ }^{29}: 15,3 \%$, con una diferencia no significativa $(\mathrm{z}=0,18, \mathrm{p}<0.05)$.

La inclusión de familiares cuidadores en el grupo control (25 participantes: 51\% de los controles), a pesar de los posibles sesgos subjetivos respecto de los cuales se arbitró un procedimiento para controlarlos, tenía la ventaja de proporcionar una mayor homogeneidad entre los enfermos y los controles Esta inclusión hacía posible también la constitución de éstos como un subgrupo de matrimonios, que permitiría contrastar los resultados de los estudios que señalaban una personalidad del cónyuge sano más dominante en relación al cónyuge enfermo.

Se ejerció para los dos grupos un control sobre algunas variables: diagnóstico, edad y sexo, cuyos datos figuran en la tabla 2.

a) Respecto al diagnóstico, en el grupo de enfermos se obtuvo de todos ellos una certificación médica de los servicios sanitarios, donde constaba el tipo de demencia y otros datos diagnósticos como el GDS de Reisberg ${ }^{30}$; en los controles, una entrevista clínica y un conocimiento a lo largo de un mínimo de 3 meses, por parte del investigador, verificó la situación de personas neurológicamente sanas, sin déficits cognitivos importantes. 
b) En relación con la edad, había una diferencia significativa $(-1,90)$ en el momento del examen, respecto al inicio de la demencia en los enfermos. Es decir, los controles, eran de más edad (2,66 años más de media).

c) En cuanto al sexo, los grupos no diferían significativamente: +/-0,22.

TABLA 2. Control de variables: GDS, edad y sexo

\begin{tabular}{|lcc|}
\hline $\begin{array}{c}\text { Grupos de enfermos } \\
n^{o}\end{array}$ & $\begin{array}{c}\text { Grupos control } \\
\%\end{array}$ & Dif. Sig \\
\hline
\end{tabular}

a) G.D.S. Reisberg

1 Ausencia déficit cognitivo 2 Déficit cognitivo muy leve

3 Déficit cognitivo leve

4 Déficit cognitivo moderado

5 Demencia inicial

6 Demencia moderada

7 Demencia severa

Fallecido

$\begin{array}{ll}. . & \ldots . . \\ . . & \ldots . . \\ . . & \ldots . . \\ . . & \ldots . . \\ 62 & 57,4 \\ 21 & 19,4 \\ 13 & 12,0 \\ 12 & 11,1\end{array}$

b) Edad

Media inicio demencia

$67,8$ años (Ds: $\pm 8,58)$
$73,3$ años (Ds: $\pm 8,28)$

$70,5$ años*(Ds: $\pm 6,92)$

70,5 años (Ds: $\pm 6,92$ )

$-1,90$

Media situación examen

$\begin{array}{cc}\text { Inicio demencia } \\ 3 & 2,8 \\ 19 & 17,6 \\ 43 & 39,8 \\ 38 & 35,2 \\ 5 & 4,6\end{array}$

Situación examen

Tramos de edad

$41-50$ años

$51-60$ años

$61-70$ años

$71-80$ años

$81-90$ años

$\begin{array}{cc}23 & 46,9 \\ 22 & 44,9 \\ 4 & 8,2 \\ . . & \ldots . . \\ . . & \ldots . . \\ . . & \ldots . . \\ . . & \ldots . . \\ . . & \ldots . .\end{array}$

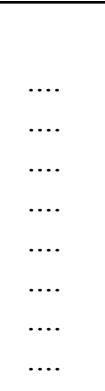

c) $\underline{\text { Sexo }}$

$\begin{array}{llllll}\text { Mujeres } & 66 & 61,1 & 29 & 59,2 & +0,22 \\ \text { Hombres } & 42 & 38,9 & 20 & 40,8 & -0,22\end{array}$

*Media de edad en la situación de examen. Ds: Desviación estándar

Además del contraste general que se realizó entre el grupo de enfermos y el grupo control para todos los factores, para algunos de éstos: muerte del padre y factores de personalidad, se realizaron también contrastes en ámbitos más específicos: Matrimonios, Edad de inicio ( $<70$ años, $\geq 70$ años), Nivel 
educativo (Analfabetos, Primarios, Secund. / Univ.) y Área Territorial (Barcelona, Baix Llobregat). En cada ámbito específico se realizaron también los contrastes según las tipologías señaladas en el ámbito 1: General. Los datos numéricos de los diferentes grupos y ámbitos se recogen en la tabla 3.

TABLA 3. Contrastes: Ámbitos general y específicos

\begin{tabular}{|c|c|c|c|c|c|}
\hline Grupos de enfermos & $\mathrm{n}^{\mathrm{o}}$ & $\%$ & Grupos control & $\mathrm{n}^{\mathrm{o}}$ & $\%$ \\
\hline \multicolumn{6}{|c|}{ Ámbito: General } \\
\hline \multicolumn{6}{|l|}{ 1: Todos los casos } \\
\hline$\overline{\text { Total Demencia }}$ & 108 & 100,0 & Total Control & 49 & 100,0 \\
\hline Total Alzheimer & 85 & 78,7 & Total Control & 49 & 100,0 \\
\hline Mujeres Alzheimer & 55 & 50,9 & Mujeres Control & 29 & 59,2 \\
\hline Hombres Alzheimer & 30 & 27,7 & Hombres Control & 20 & 40,8 \\
\hline Demencia Mixta-Vascular & 17 & 15,7 & Total Control & 49 & 100,0 \\
\hline \multicolumn{6}{|c|}{ Ámbitos especificos } \\
\hline 2: Matrimonios & & & & & \\
\hline Enfermo & 24 & 22,2 & Cónyuge no enfermo & 24 & 48,9 \\
\hline \multicolumn{6}{|l|}{ 3: $\underline{\text { Inicio precoz y tardío }}^{*}$} \\
\hline$\overline{<\text { de } 70 \text { años }}$ & 57 & 52,7 & $<$ de 70 años & 17 & 34,6 \\
\hline$\geq$ de 70 años & 51 & 47,2 & $\geq$ de 70 años & 32 & 65,3 \\
\hline \multicolumn{6}{|l|}{ 4: Nivel educativo } \\
\hline$\overline{\text { Analfabetos }}$ & 28 & 25,9 & Analfabetos & 5 & 10,2 \\
\hline Primarios & 54 & 50,0 & Primarios & 17 & 34,6 \\
\hline Secund. y Univers. & 23 & 21,2 & Secund. y Univers. & 27 & 55,1 \\
\hline \multicolumn{6}{|l|}{ 5: Área Territorial } \\
\hline Barcelona ciudad & 32 & 29,6 & Barcelona ciudad & 35 & 71,4 \\
\hline Baix Llobregat & 76 & 70,3 & Baix Llobregat & 14 & 28,5 \\
\hline
\end{tabular}

* En los grupos control la edad es la de la situación de examen

\section{Material}

El material básico en la investigación fue el cuestionario confeccionado de forma expresa para la recogida de datos. Las entrevistas previas desarrolladas durante los años 1992-96, con los familiares de los enfermos de Alzheimer, permitieron plasmar aquellos conceptos que aparecían más relevantes en la vida de los enfermos antes de contraer la enfermedad; añadiéndose también aquellos factores de 
riesgo reseñados en las investigaciones publicadas. La elaboración de un cuestionario permitía unificar en un sólo documento todas las observaciones que se querían realizar. Previamente se realizaron diez ensayos para comprobar su operatividad, su validez, al contrastar la información obtenida con dos familiares y su fiabilidad, realizando los mismos exámenes con dos entrevistadores, siendo los resultados satisfactorios.

Se procuró que el cuestionario fuese una entrevista lo más estructurada posible con el objetivo de recoger todos los datos y al mismo tiempo minimizar la posibilidad de los sesgos subjetivos. En todos los ítems, las posibles respuestas están predeterminadas mediante casillas, aunque también se incluye la posibilidad de recoger otras, o bien observaciones que permitan su clarificación. En otros ítems la respuesta es abierta, cuando hay que determinar las edades, la clase de eventos vitales, la clase de trabajo, el tipo de estudios, etc. ya que la variabilidad es demasiado grande como para permitir una codificación previa. Finalmente en otros ítems, por ejemplo en los factores de personalidad, el entrevistador debía codificar la respuesta en función de lo expresado por el entrevistado.

El tiempo dedicado la realización de cada entrevista fue de 60-70 minutos. Los ítems del cuestionario en la tabla 4, permiten ver al mismo tiempo el conjunto de las variables y factores que se manejaron.

TABLA 4. Ítems del cuestionario

1a) Datos generales (Grupo enfermos)

- Edad, sexo, parentesco cuidador, diagnóstico, institución, profesional, año 1er. diag., años evolución, G.D.S.

- Autonomía actual: comer, vestirse, higiene, lenguaje, reconocimiento, movilidad

1b) Datos generales (Grupo control)

- Persona mayor: edad, sexo, taller memoria - Cuidador principal: edad, sexo, parentesco enfermo, grupo apoyo

- Estado de salud actual: grado de calificación, tipo de enfermedad y grado de afectación

2) Antecedentes de enfermedades físicas importantes (personales y familiares)

- Tipo de enfermedad, parentesco familiar, edad de la persona que la padece

- Familiares con demencia: tipo de demencia y clase de familiar

- Golpe en la cabeza: circunstancias, edad en la que ocurre el golpe 
TABLA 4. Ítems del cuestionario (continuación)

3) Antecedentes de trastornos psíquicos (personales y familiares)

- Tipo: neurosis (obsesiones, fobias, histeria), depresiones (distimia, mayor), psicosis (esquizofrenia, paranoia)

- Edad en la que aparece el trastorno

- Tratamientos: farmacológico, psicológico

4) Gráfico familiar de ler grado (hermanos, padres, tíos y abuelos)

- Edades actuales o de muerte y enfermedades importantes

5) Estilo y ambiente familiar paterno.

- Estilo educativo general: autoritario, democrático, permisivo y de rechazo

- Rasgos personalidad del padre y de la madre: autoritario, afectuoso y ausente

- Edad del enfermo cuando murieron el padre y la madre

6) Eventos vitales en Infancia y Adolescencia, Juventud y Mediana Edad, Vejez (personales y familiares)

- Tipo: clase de evento

- Reacción frente al evento: ansiedad - depresión - reacción psicosomática

- Grado afectación: mucho, bastante, poco

- Edad en el que ocurrió el evento

7) Nivel educativo

- Nivel: analfabeto, primarios, secundarios, universitarios

- Tipo de estudios

- Edad final de escolarización

8) Ocupación laboral

- Clase: nombre del trabajo

- Tipo: ama de casa, manual, campo, administrativo, servicios

- Contratación: autónomo, asalariado, funcionario

- Nivel: trabajador, especialista, directivo medio, alto directivo

9) Actividades y relaciones sociales en la vida adulta y Vejez

- Tipo de actividad: entidades, vecinos, compañeros de trabajo, amistades, sólo familia

- Grado de relación: mucho, bastante, poco, nada

10) Relaciones de pareja o de vínculos afectivos principales (15 años antes de la enfermedad)

- Calificativos relación de pareja: muy buena (1), buena (2), normal (3), mala (4), muy mala (5)

11) Perfiles de personalidad y relación (15 años antes de la enfermedad). Escala bipolar

- Grado: Mucho (1), Bastante (2), Poco (3), Indefinición (4), Poco (5) Bastante (6), Mucho (7)

* Factor I: Energía

Autónomo / Dependiente

Dominante / Sumiso

Crit. propio / Imitador

Seguro / Inseguro

Afrontador / Evitador

Asertivo / Inhibido

Relación de pareja

Autónomo / Dependiente

Dominante / Sumiso
* Factor II: Afabilidad

Generoso / Egoísta

Altruista / Egocéntrico

Extrovertido / Introvertido

Sociable / Retraído

* Factor III: Tesón

Responsable / Irresponsable

Organizado / Caótico

Realista / Utópico

Convencional / Anárquico
* Factor IV: Estabilidad Emocional Optimista / Pesimista

Entero / Afectado

Sereno / Ansioso

Impulsivo / Contenido

Pacífico / Irritable

* Factor V: Apertura Mental

Interesado / Indiferente

Flexible / Rígido 
En relación a los perfiles de personalidad y relación se trataba, en cada uno de los 23 elementos dicotómicos, seleccionados según el criterio del autor en función de lo detectado en las entrevistas previas, de situar las características de los participantes en una escala bipolar con siete grados, previa explicación de cada concepto y situando la descripción de los participantes en un tiempo 15 años anterior a la enfermedad. A modo de ejemplo, para el primer elemento bipolar Extroversión / Introversión, se preguntaba: ¿En que medida la persona era proclive a explicar sus sentimientos y vivencias personales a los demás, o no? En función de la respuesta el entrevistador debía codificarla en alguno de los siete grados posibles: mucho: 1, bastante: 2 y un poco: 3, para el polo Extroversión; indefinición: 4; un poco: 5, bastante: 6 y mucho: 7, para el polo Introversión.

Se consideró interesante para el posterior análisis, agrupar los elementos siguiendo los criterios teóricos de los cinco grandes factores de personalidad, explicitados en el Cuestionario Big Five ${ }^{31}$ (en el NEO-PI ${ }^{32}$ son sensiblemente diferentes), sin aplicar no obstante su planteamiento metodológico para el examen.

Factores de personalidad Criterios

I Energía:

Capacidad para enfrentarse a las dificultades y resolverlas autónomamente

II Afabilidad:

Capacidad de apertura, entendimiento y cooperación con los demás

III Tesón:

Capacidad de organización y responsabilidad

IV Estabilidad emocional:

Capacidad de control y elaboración ante situaciones amenazantes

V Apertura mental:

Capacidad de interesarse por temas no familiares y tener nuevas experiencias

\section{Procedimiento}

Las entrevistas se desarrollaron durante el período 1997-99. Con las personas del grupo de enfermos se realizaron a través del familiar cuidador con el que el enfermo había tenido el vínculo más intenso; en cambio, con los participantes de los grupos control, las entrevistas se realizaron directamente, ya 
que se consideró que ellos mismos tenían la mayor información sobre sus propias vidas. No obstante, la información recogida fue contrastada con la recibida de otros familiares, constituyéndose un grupo 2 de enfermos con 6 familiares y un grupo 2 de control con 4 familiares, con el objetivo de controlar la fiabilidad de la información de los grupos 1. El resultado de este contraste fue la no detección de contradicciones importantes, aunque sí se constataron lagunas de información en los grupos 2, en relación a eventos vitales, fundamentalmente de la infancia.

En la realización de las entrevistas participaron alumnos de $4^{\circ}$ curso de la Licenciatura de Psicología de la Universidad de Barcelona. Recibieron entrenamiento previo e instrucciones escritas para la realización de la entrevista durante 3 sesiones de 90 minutos con un seguimiento posterior con resultados satisfactorios. Cada entrevista realizada por los alumnos fue supervisada personalmente, comentando y resolviendo con el entrevistador las dudas o dificultades que se suscitaron. Hacer constar que un alto porcentaje de las entrevistas (60\%) habían sido realizadas anteriormente por el autor de la investigación, con un intervalo de tiempo de 6-12 meses, contrastando la información obtenida en las dos situaciones y verificando su fiabilidad.

Se calcularon las diferencias significativas entre los valores del grupo de enfermos y el grupo control: puntuaciones $\mathrm{z}$ para las proporciones y puntuaciones $\mathrm{t}$ para las medias. La comparación de proporciones se realizó aplicando la prueba $\mathrm{z}^{26}$, mientras que en la comparación de medias se utilizó la prueba t para muestras independientes del programa SPSS. Se determinó un nivel alfa de 0.05 para todas las pruebas estadísticas $(\mathrm{p}<0.05)$. Los contrastes estadísticos realizados pueden ser considerados como unilaterales o direccionales ${ }^{26-28}$, con puntuaciones significativas $>1,65$ para $p<0.05$ y puntuaciones $>2,33$ para $\mathrm{p}<0.01$. 


\section{Resultados}

Debido a la extensión del estudio, los resultados son una selección de los más significativos; la globalidad de los datos puede consultarse en el estudio completo ${ }^{1}$. En las figuras y tablas se muestra la comparación entre los grupos globales: Total Demencia (TD), Total Alzheimer (TA), Demencia Mixta-Vascular (DMV), y los grupos control: Total Control (TC) y Total Población (TP). Se hacen constar también los contrastes entre grupos internos cuando sean significativos: Mujeres Alzheimer (MA), Hombres Alzheimer (HA) y Mujeres Control (MC), Hombres Control (HC).

En los contrastes de los grupos globales (TD, TA) se ha utilizado un criterio restrictivo: además de tener una diferencia significativa, las diferencias de sus grupos internos (MA, HA y DMV) han de ser del mismo signo y al menos superiores a 1,00. Las diferencias significativas del texto escritas en normal son $>1,65(\alpha 0,05)$ y las negritas son $>2,33(\alpha \mathbf{0 , 0 1})$.

Aunque el título del artículo se refiere a los enfermos con demencia, los resultados tienen una mayor validez estadística para los grupos Alzheimer, ya que en éstos los tamaños de las muestras son $\geq 30$ : TA, $n=85 ;$ MA, $n=55 ;$ HA, $n=30$; mientras que en el D. Mixta y Vascular es $<30$ : DMV, $n=17$.

En el texto, los datos irán precedidos de la diferencia significativa y entre paréntesis los grupos contrastados con sus proporciones, Ej.: +2,26 (TD: 34,3\% / TC: 18,1\%); en el contraste de medias se

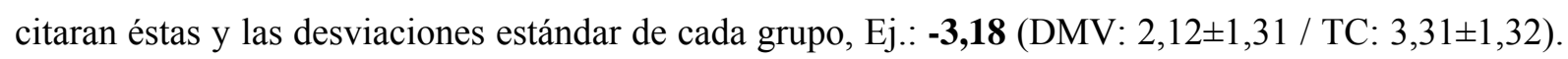
El número de participantes de cada grupo figura en la tabla 3.

\section{Manifestaciones psíquicas y/o psicosomáticas familiares y personales}

1.1 Antecedentes familiares de trastornos psíquicos (Fig. 1). En el conjunto de los enfermos de Demencia, aparecen los trastornos graves sin especificar (se incluyen los que los familiares relataban 
como graves, con ingresos psiquiátricos, aunque sin precisar el diagnóstico): +2,71 (TD: 6,4\% / TC 0,0\%), los suicidios: $+2,03$ (TD: 3,7\% / TC: 0,0\%) y las psicosis: $+1,67$ (TD: 7,4\% / TC: 2,0\%), en este caso con diagnósticos explícitos por parte de los familiares de esquizofrenia o paranoia. A pesar de que estos porcentajes son pequeños, superan ampliamente los índices normativos para el conjunto de la población. En el grupo Total Alzheimer destaca la mayor presencia de total de trastornos psíquicos familiares: +3,61 (TA: 40,6\% / TC: 14,2\%), la mayor ansiedad: $+2,30$ (TA: 5,9\% / TC: 0,0\%) y las drogas: $+1,76$ (TA: 3,6\% / TC: 0,0\%). El grupo Demencia Mixta-Vascular es el de menor patología psíquica familiar, sin que aparezca ningún trastorno significativo.

Subrayar que los trastornos depresivos (TD: 11,1\% / TC: 12,2 \%) y el retraso mental (TD: 1,9\%, TC: $0,0 \%)$ en los familiares, no presentan diferencias significativas.

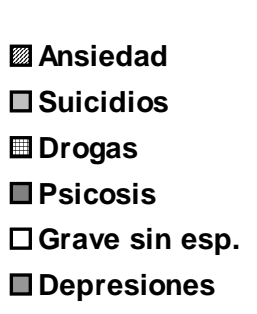

- Total psíquicos

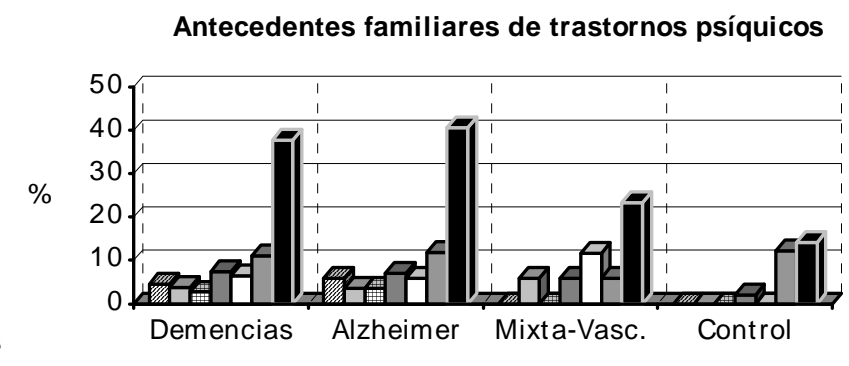

Figura 1. Antecedentes familiares de trastornos psíquicos. Comparación de los grupos de enfermos: Total Demencia, Total Alzheimer, Demencia Mixta-Vascular, con el grupo Total Control

1.2 Antecedentes personales de trastornos psíquicos. Los datos más relevantes son la menor presencia de trastornos depresivos: -2,54 (TD: 29,6\% / TC: 50,9\%) en el grupo de enfermos de Demencia, así como un menor número de tratamientos y fármacos, en Mujeres Alzheimer: -2,34 (MA: 29,1\% / MC: 55,1\%). En Hombres Alzheimer, sí aparecen los trastornos derivados del abuso del alcohol: $+1,82$ (HA: 10,0\% / HC: 0,0\%). 
1.3 Repuestas psicosomáticas a eventos vitales (Fig. 2). Existe una mayor respuesta psicosomática generalizada para los enfermos de Demencia: $+2,26$ (TD: $34,3 \%$ / TC: $18,1 \%$ ), con una mayor presencia de caídas y hernias discales para el grupo Mujeres Alzheimer: +2,59 (MA: 10,9 \% / MC: $0,0 \%)$ y con una mayor respuesta cardio-cerebro vascular para los grupos Hombres Alzheimer: $+2,13$ (HA: 13,2\% / HC: 0,0\%) y Demencia Mixta-Vascular: +1,90 (DMV: 17,6\% / TC: 4,0\%).
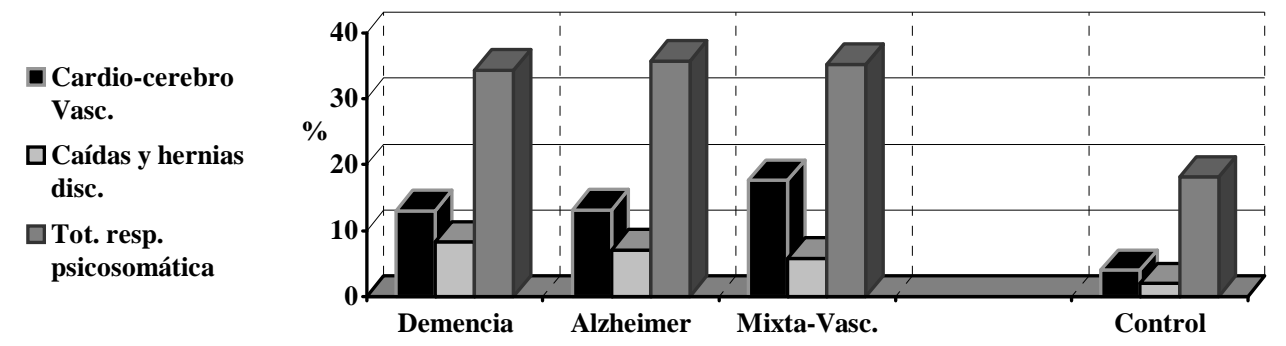

Figura 2. Respuestas psicosomáticas a eventos vitales. Comparación de los grupos de enfermos: Total Demencia, Total Alzheimer, Demencia Mixta-Vascular, con el grupo Total Control

\section{Familia y estilo educativo}

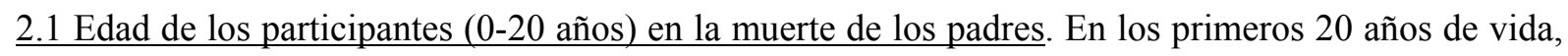
en el grupo de enfermos de demencia, se produce un mayor número de pérdidas del padre: +2,83 (TD: 36,2\% / TC: 16,3\%), mayor en el grupo Mujeres Alzheimer: +3,04 (MA: 41,8\% / MC: 13,7\%), así como un menor número de pérdidas de la madre, especialmente en el grupo Hombres Alzheimer: 1,72 (HA: 6,7\% / HC: 25,0\%). La muerte del padre y de la madre, conjuntamente en el mismo período, aparece asimismo como significativa en el grupo Total Alzheimer: +1,73 (TA: 8,3\% / TC: $2,0 \%)$.

$\underline{2.2 \text { Comparación con el total de la población de la edad de la persona }(0-20 \text { años }) \text { en la muerte de los }}$ padres. (Fig. 3). Los datos de la población general ${ }^{29}$, refuerzan las observaciones realizadas en el grupo 
control. En los menores de 70 años se producen más pérdidas del padre antes de los 20 años en todos los grupos de enfermos: +3,46 (TD: 35,1\% / TP: 13,2\% / TC: 17,7\%). En los mayores de 70 años, la muerte del padre sólo es significativa para el grupo Total Alzheimer: +2,87 (TA: 42,1\% / TP: 19,1\% / TC: $15,7 \%$ ). De forma global, la pérdida del padre es significativa para todos los grupos: $+\mathbf{4 , 5 0}$ (TD: 36,1\% / TP: 15,3\% / TC: 16,3\%); la muerte de la madre y padre conjuntamente sólo lo es para Mujeres Alzheimer: +1,67 (MA: 9,1\% / TP: 2,6\% / TC: 2,0\%). El grupo con más pérdidas, tanto en el contraste con la población como con los controles, es Mujeres Alzheimer.
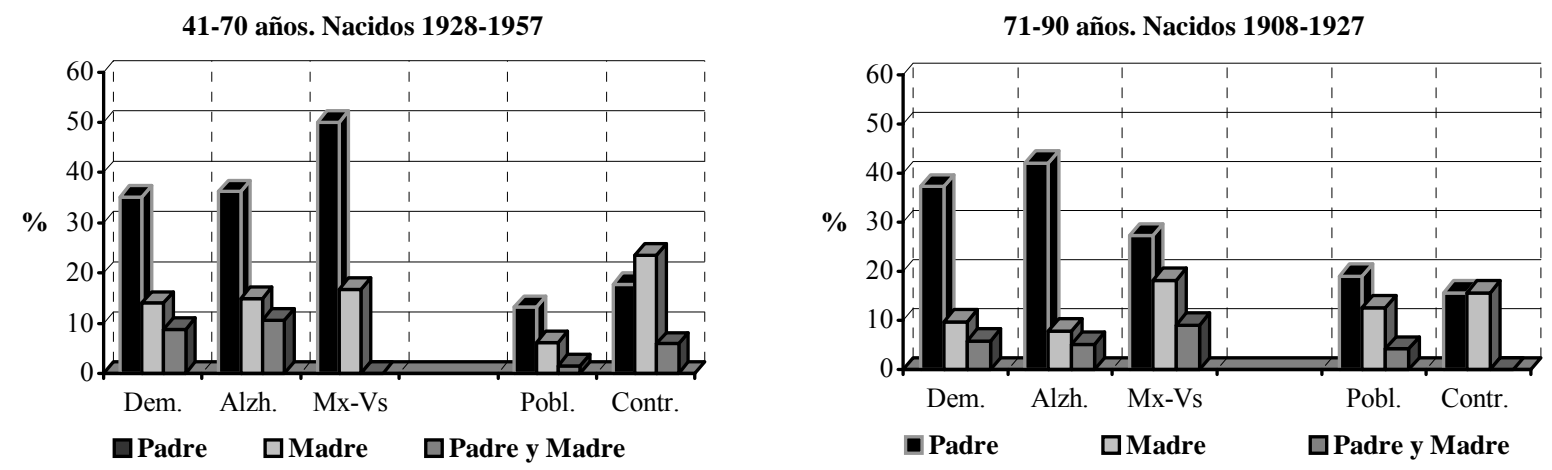

Figura 3. Edad de los participantes (0 - 20 años) en la muerte de los padres, según períodos de nacimiento: 1908 -1927 y 1928 1957. Comparación de los grupos de enfermos: Total Demencia, Total Alzheimer, Demencia Mixta-Vascular con el Total de la población y el grupo Total Control

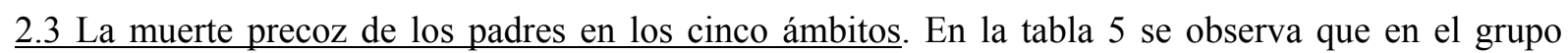
Mujeres Alzheimer se concentran el mayor número de pérdidas del padre en la mayoría de los ámbitos analizados, de manera significativa: General (MA: 41,8\% / MC: 13,7\%), Matrimonios (MA: 44,4\% / MC: 0,0\%), menores de 70 años (MA: 44,8\% / 10,0\%), mayores de 70 años (MA: 38,5\% / MC: 15,8\%), Analfabetos (MA: 27,8\% / MC: 0,0\%), Primarios (MA: 50,0\% / MC: 18,2\%), Barcelona (MA: 71,4\% / MC: 13,0\%); sólo no son significativos Secund.-Univ.: +0,24 (MA: 16,7\% / MC: 12,5\%) y Baix Llobregat: +0,60 (MA: 31,7\% / MC: 20,0\%). 
Se constata también la correlación entre bajo nivel educativo y muerte del padre en todos los grupos, no presentándose ésta en el nivel educativo superior, Sec.-Un.: -0,11 (TD: 21,7\% / TC 23,1\%).

En el Área Territorial, Baix Llobregat: +1,04 (TD: 34,2\% / TC: 21,4\%) no tiene diferencias significativas en ningún grupo, ya que los porcentajes de muerte de los padres están más igualados, contrastando con las grandes diferencias de Barcelona (TD: 40,6\% / TC: 14,7\%).

Finalmente, la muerte de la madre es menor de forma general en Hombres Alzheimer (HA: 6,7\% /

HC: 25,0\%) y en algunos ámbitos del grupo Demencia Mixta-Vascular.

TABLA 5. Diferencias significativas en la muerte de los padres, antes de los 20 años de edad de los participantes

\begin{tabular}{|c|c|c|c|c|c|c|c|c|c|c|}
\hline \multirow[t]{2}{*}{ Ámbitos } & \multirow{2}{*}{$\begin{array}{l}\text { Muerte } \\
\text { padres }\end{array}$} & \multirow{2}{*}{$\begin{array}{l}\text { 1) Todos } \\
\text { los casos }\end{array}$} & 2) Matr. & \multicolumn{2}{|c|}{ 3) Edad inicio } & \multicolumn{3}{|c|}{ 4) Nivel educativo } & \multicolumn{2}{|c|}{ 5) Área Territ. } \\
\hline & & & Enf.-Con. & $<70 a$ & $\geq 70 a$. & Analf. & Prim. $\mathrm{Se}$ & $-U n$. & $B C N$ & Baix Ll. \\
\hline Total & Padre & $+2,82 * *$ & $+1,12$ & $+1,43$ & $+2,32 *$ & $+3,64 * *$ & $+2,80$ ** & $-0,11$ & $+2,45 * *$ & $+1,04$ \\
\hline \multirow[t]{2}{*}{ Demencia } & Madre & $-0,99$ & $-1,12$ & $-0,84$ & $-0,75$ & $-0,49$ & $-1,55$ & $+0,20$ & $+0,14$ & $-1,44$ \\
\hline & Total & $+1,62$ & 0,00 & $+0,49$ & $+1,47$ & $+1,13$ & $+0,77$ & $+0,03$ & $+2,34 * *$ & $-0,36$ \\
\hline Total & Padre & $+3,01 * *$ & $+1,40$ & $+1,47$ & $+2,58 * *$ & $+3,61 * *$ & $+2,94 * *$ & $+0,25$ & $+2,75 * *$ & $+1,12$ \\
\hline \multirow[t]{2}{*}{ Alzheimer } & Madre & -0.99 & $-1,03$ & $-0,74$ & $-1,00$ & $-0,84$ & $-1,34$ & $+0,20$ & $+0,31$ & $-1,50$ \\
\hline & Total & $+1,84 *$ & $+0,39$ & $+0,62$ & $+1,63$ & $+0,81$ & $+1,04$ & $+0,37$ & $+2,89 * *$ & $-0,32$ \\
\hline Mujeres & Padre & $+3,04 * *$ & $+2,68 * *$ & $+2,63 * *$ & $+1,78^{*}$ & $+2,64 * *$ & $+2,12 *$ & $+0,24$ & $+4,20 * *$ & $+0,60$ \\
\hline \multirow[t]{2}{*}{ Alzheimer } & Madre & $+0,10$ & $+0,11$ & $+0,88$ & $-0,82$ & 0,00 & $-0,02$ & $+1,03$ & $+1,16$ & $-0,55$ \\
\hline & Total & $+2,71 * *$ & $+2,30^{*}$ & $+2,95 * *$ & $+1,01$ & $+2,64 * *$ & $+1,85^{*}$ & $+1,12$ & $+8,60 * *$ & $+0,06$ \\
\hline Hombres & Padre & $+1,08$ & $-0,41$ & $-0,32$ & $+1,97^{*}$ & $+2,00 *$ & $+2,67 * *$ & $-0,30$ & $-0,45$ & $+1,18$ \\
\hline \multirow[t]{2}{*}{ Alzheimer } & Madre & $-1,72 *$ & $-2,36 * *$ & $-1,91 * *$ & $-0,55$ & 0,00 & $-1,93 *$ & $-1,06$ & $-1,56$ & $-1,40$ \\
\hline & Total & $-0,35$ & $-0,81$ & $-2,17 * *$ & $+1,43$ & $+1,63$ & $-0,62$ & $-0,74$ & $-1,41$ & $-0,15$ \\
\hline Demencia & Padre & $+1,49$ & $+0,12$ & $+1,38$ & $+0,79$ & $+1,82 * *$ & $+4,08 * *$ & $-0,74$ & $+0,62$ & $+1,16$ \\
\hline \multirow[t]{2}{*}{ Mixta-Vasc. } & Madre & $-0,05$ & $-0,67$ & $-0,37$ & $+0,19$ & $+0,70$ & $-2,66 * *$ & $+0,10$ & $-0,13$ & $-0,34$ \\
\hline & Total & $+1,31$ & $-0,45$ & $+0,99$ & $+0,83$ & $+2,37 * *$ & $+0,75$ & $-0,51$ & $+0,45$ & $+0,80$ \\
\hline
\end{tabular}

Matrim.: Matrimonios, Enf.: Enfermo, Con.: Cónyuge, Territ.: Territorial, Analf.: Analfabetos, Prim.: Estudios Primarios, Sec.-Un.: Estudios secundarios y universitarios, BCN: Barcelona ciudad, Baix L1.: Comarca del Baix Llobregat.

Sólo se consignan las diferencias significativas: $* \mathrm{p}<0.05 ; * * \mathrm{p}<0.01$.

Cursiva: grupos internos (MA, HA o DMV) no significativos, aunque del mismo signo y superiores a 1,00, en grupos globales (TD o TA) con diferencia significativa 


\section{Educación, trabajo y relaciones sociales}

3.1 Nivel educativo (Fig. 4). Un menor nivel educativo, con una importante tasa de analfabetismo, aparece como lo más destacado, especialmente para el grupo Mujeres Alzheimer: +4,33 (MA: 25,5\% / MC: 0,0\%) y el Demencia Mixta-Vascular: +2,44 (DMV: 29,4\% / TC: 2,0\%). Asimismo en el grupo Total Alzheimer se da una menor tasa de estudios universitarios: -3,03 (TA: 1,2\% / TC: 18,4\%).

3.2 Ocupación laboral. En cuanto al tipo de trabajo (Fig. 4), mayor dedicación de Mujeres Alzheimer a labores de la casa: $+2,15$ (MA: $58,2 \%$ / MC: $34,4 \%$ ) y menor presencia de trabajos cualificados, para el total del grupo Alzheimer: -2,69 (administrativos y prof. liberales, TA: 12,0\% / TC: 32,3\%), y para Demencia Mixta-Vascular: -3,69 (administrativos, DMV: 0,0\% / TC: 20,3\%), es lo más notable.

Referente a la contratación, lo más destacable es una menor cualificación: menos autónomos para todos los grupos: -2,39 (TD: 13,0\% / TC: 30,6\%), menos funcionarios para Total Alzheimer: -2,37 (TA: 0,0\% / TC: 10,2\%) y más ninguna contratación, igualmente en Mujeres Alzheimer: +4,31 (MA: 34,6\% / MC: 3,4\%) y Demencia Mixta-Vascular: +2,83 (DMV: 35,2\% / TC: 2,0\%). Tanto en los temas educativos, como en los laborales, el grupo Hombres Alzheimer tiene un menor déficit.
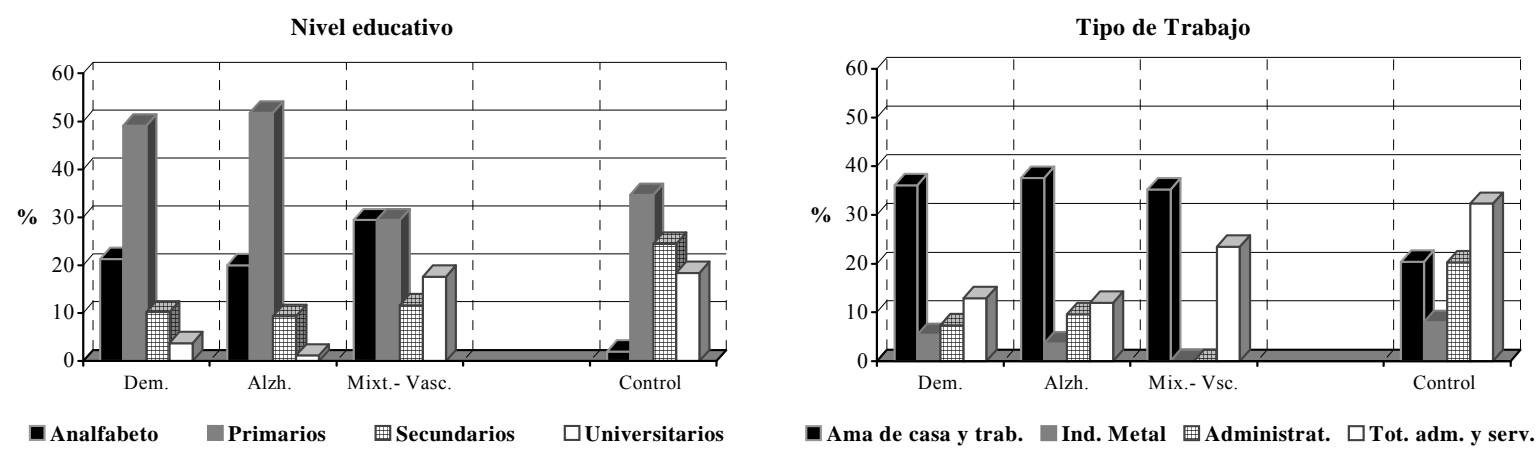

Figura 4. Nivel educativo y Tipo de trabajo. Comparación de los grupos de enfermos: Total Demencia, Total Alzheimer, Demencia Mixta-Vascular con el grupo Total Control 
3.3 Relaciones sociales. Aunque el grado de relación social es menor en todos los enfermos de Demencia, excepto en el de Hombres Alzheimer, las diferencias no son significativas. Es en el grupo Demencia Mixta-Vascular, donde la menor sociabilidad, aparece con mayor rotundidad: -3,18 (DMV: $2,12 \pm 1,31$ / TC: $3,31 \pm 1,32$ ); en este grupo se darán también las mayores cotas de retraimiento en la temática de personalidad.

En cuanto al tipo de relaciones, están más centrados en la familia, el grupo Mujeres Alzheimer: +3,21 (MA: 32,1\% / MC: 6,9\%) y el grupo Demencia Mixta-Vascular: +2,69 (DMV: 47,0\% / TC: 12,2\%). Estos dos grupos tienen también una menor participación en Entidades, MA: -3,15 (MA: 67,8\% / MC: 92,7\%) y DMV: -3,12 (DMV: 46,9\% / TC: 87,5\%).

\section{Eventos vitales}

4.1 Temática personal en el conjunto del ciclo vital (Fig. 5). Viendo el conjunto de eventos vitales en todas las etapas, la temática dominante, en el área personal, es la de menor guerra (eventos vitales relacionados con la guerra civil española 1936-39) para todos los grupos de enfermos de Demencia: -1,88 (TD: 30,8\% / TC: 46,6\%). En el grupo Mujeres Alzheimer están presentes las dificultades económicas: $+2,04$ (MA: 27,1\% / MC: 10,1\%), así como un menor cómputo de eventos personales: -2,33 (MA: 108,2\% / MC: 143,9\%) y en el grupo Hombres Alzheimer, destacan los eventos laborales: +3,69 (HA: 86,5\% / HC: 40,0\%). El grupo Demencia Mixta-Vascular es el que tiene más eventos personales estresantes, destacando la enfermedad propia: +2,86 (DMV: 46,9\% / TC: 10,1\%) y el total de conflictos personales: $\mathbf{+ 8 , 5 8}$ (DMV: 105,4\% / TC: 30,3\%). 
4.2 Temática familiar en el conjunto del ciclo vital. (Fig. 5). En los temas familiares se constata un menor índice de enfermedades de familiares (debido a que el 51\% de los controles son cuidadores de enfermos) en todos los grupos de enfermos de Demencia: -5,62 (TD: 32,4\% / TC: 75,3\%).

Por otra parte, en todos los grupos de enfermos se da globalmente un mayor número de problemas familiares (discusiones familiares, dificultades emancipación hijos, separaciones familiares): $+\mathbf{5 , 4 5}$ (TD: 100,4\% / TC: 62,6\%), en Hombres Alzheimer, la muerte del hermano: +2,29 (HA: 26,6\% / HC: 5,0\%) y en Mujeres Alzheimer, la muerte del esposo: +1,75 (MA: 29,1\% / MC: 13,6\%).

Eventos vitales: Temática personal

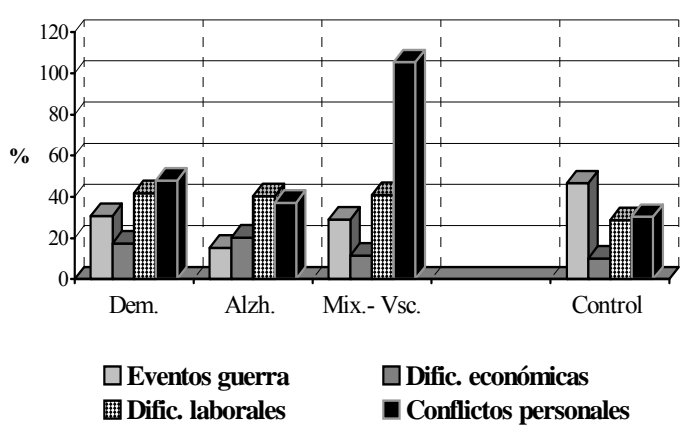

Eventos vitales: Temática familiar

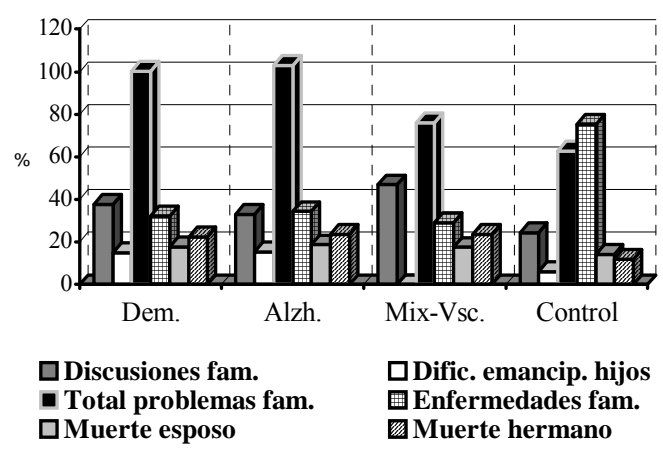

Figura 5. Eventos vitales: Temática global personal y familiar. Comparación de los grupos de enfermos: Total Demencia, Total Alzheimer, Demencia Mixta-Vascular con el grupo Total Control

$\underline{4.3}$ Eventos vitales vinculados al inicio de demencia (5 últimos años). En el área personal sólo son significativos el mayor número de enfermedades propias para el grupo Demencia Mixta-Vascular: +2,42 (DMV: 64,7\% / TC: 32,3\%). En el área familiar, excluyendo las enfermedades familiares, constatar para Mujeres Alzheimer, la muerte del esposo: +2,82 (MA: 12,7\% / MC: 0,0\%) y las dificultades en la emancipación de los hijos: +2,59 (MA: 10,9\% / MC: 0,0\%); y para Hombres Alzheimer, la muerte de la madre: +2,87 (HA: 33,4\% / HC: 5,0\%) y la muerte del hermano: $+1,82$ (HA: 10,0\% / HC: 0,0\%). 


\section{Perfiles de personalidad y relación}

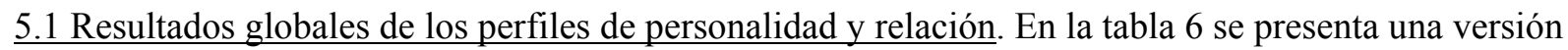
simplificada, contabilizando sólo las diferencias significativas de las medias aritméticas del grupo de enfermos superiores a 3,00, suprimiendo los aspectos con las puntuaciones más bajas.

Considerando globalmente los datos, lo más notable para todos los grupos de enfermos es el mayor

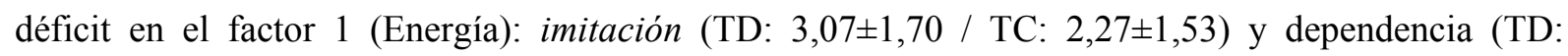

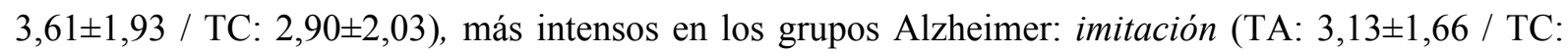

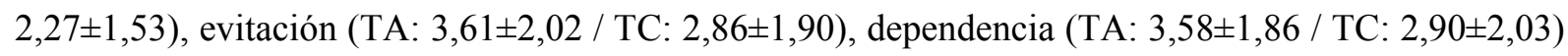

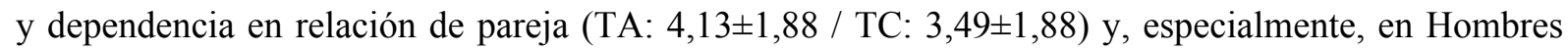

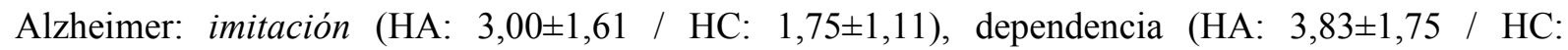

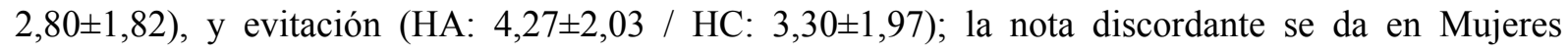

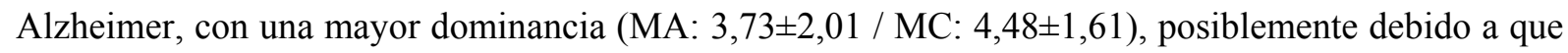
el grupo control se puntuó como muy sumiso.

En el grupo Demencia Mixta-Vascular el déficit del factor II (Afabilidad) es más intenso: retraimiento

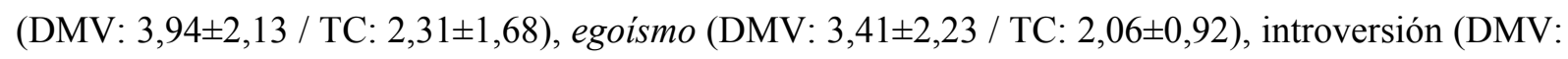

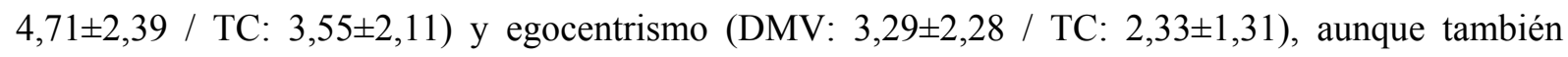

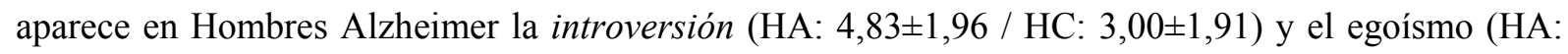

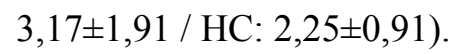

En todos los grupos de enfermos se da, en el factor IV (Estabilidad emocional), el pesimismo (TD:

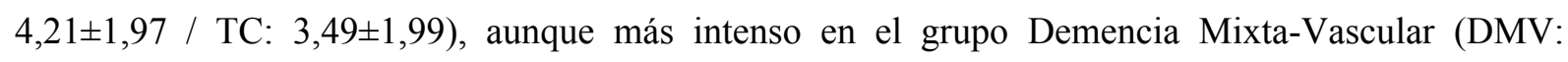

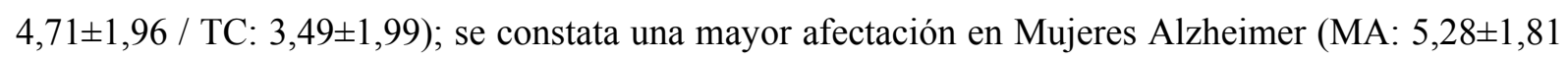


( MC: 4,41 $\pm 2,12$ ), mientras que Demencia Mixta-Vascular aparece con mayor entereza (DMV: $3,24 \pm 1,98$ / TC: $4,31 \pm 2,23)$.

En el factor V (Apertura Mental), se da un importante déficit, en Mujeres Alzheimer, en indiferencia:

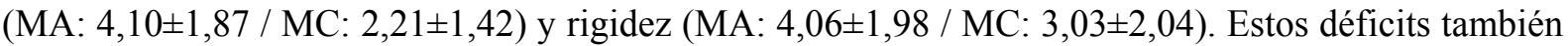
aparecen en la Demencia Mixta y Vascular, aunque invertidos en su intensidad: rigidez (DMV: 4,71 $\pm 2,14$ / TC: $3,16 \pm 2,00$ ) e indiferencia (DMV: 3,82 $\pm 2,24$ / TC: $2,67 \pm 1,93$ ).

El factor III (Tesón) es el que aparece con menor déficit en todos los grupos, únicamente en Hombres

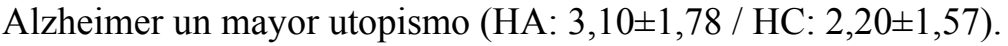

TABLA 6. Perfiles de personalidad y relación

Diferencias significativas de medias aritméticas superiores a 3,00

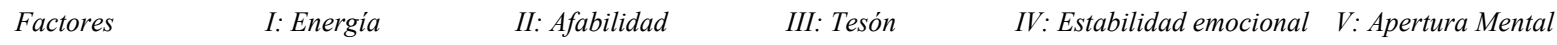

\begin{tabular}{|c|c|c|c|c|c|c|c|c|c|c|}
\hline $\begin{array}{l}\text { Total } \\
\text { Demencia }\end{array}$ & $\begin{array}{l}\ldots . . \\
\ldots . .\end{array}$ & $\begin{array}{l}\text { / Imit. } \\
\text { / Dep. }\end{array}$ & $\begin{array}{l}2,81 * * \\
2,09^{*}\end{array}$ & $\begin{array}{lll}\ldots . & / & \ldots . \\
\ldots . & / & \ldots .\end{array}$ & & $\begin{array}{l}\ldots \ldots / \ldots \ldots \\
\ldots \ldots . / \ldots \ldots\end{array}$ & $\begin{array}{l}\ldots . . / \text { / Pesim. } \\
\ldots . / \text { / .... }\end{array}$ & $2,10^{*}$ & $\begin{array}{ll}\ldots . . & / \\
\ldots . . & \ldots \\
\ldots . & \ldots\end{array}$ & \\
\hline $\begin{array}{l}\text { Total } \\
\text { Alzheimer }\end{array}$ & $\begin{array}{l}\cdots \cdots \\
\cdots . . \\
\cdots \cdots \\
\cdots . .\end{array}$ & $\begin{array}{l}\text { / Imit. } \\
\text { / Evit. } \\
\text { / Dep. } \\
\text { / Dep (r) }\end{array}$ & $\begin{array}{l}2,97^{* *} \\
2,11^{*} \\
1,96^{*} \\
1,89^{*}\end{array}$ & $\begin{array}{lll}\ldots \ldots & / & \ldots \\
\ldots \ldots & / & \ldots \\
\ldots . & \\
\ldots \ldots & / & \ldots . \\
\ldots \ldots & / & \ldots .\end{array}$ & & $\begin{array}{l}\ldots . . \\
\ldots \ldots \\
\ldots \ldots .\end{array}$ & $\begin{array}{l}\ldots . . / \text { Pesim. } \\
\ldots \ldots . / \ldots \ldots \\
\ldots \ldots . / \ldots . . \\
\ldots . . / \ldots .\end{array}$ & 1,53 & $\begin{array}{lll}\ldots . & \ldots \\
\ldots . & \ldots & \ldots . \\
\ldots \ldots & \ldots \ldots \\
\ldots . & \ldots \ldots \\
\ldots . / & \ldots \ldots\end{array}$ & \\
\hline $\begin{array}{l}\text { Mujeres * * } \\
\text { Alzheimer }\end{array}$ & $\begin{array}{c}* 1,87 \text { Dom }(\mathrm{r}) \\
\ldots . . \\
\ldots . . \\
\ldots . \\
\ldots . .\end{array}$ & $\begin{array}{l}\text { / ..... } \\
\text { / Evit. } \\
\text { / Imit. } \\
\text { / Dep.(r) } \\
\text { / Dep. }\end{array}$ & $\begin{array}{l}1,54 \\
1,49 \\
1,36 \\
1,03\end{array}$ & 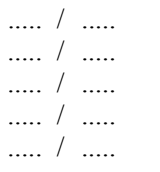 & & $\begin{array}{ll}\ldots . . & / \ldots \ldots \\
\ldots . . & / \ldots \ldots \\
\ldots . . & / \ldots . \\
\ldots . . & / \ldots \ldots \\
\ldots \ldots . & / \ldots . .\end{array}$ & 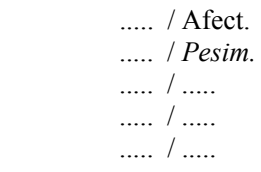 & $\begin{array}{l}1,80^{*} \\
0,95\end{array}$ & 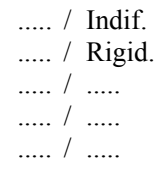 & $\begin{array}{l}5,10^{* *} \\
2,21^{*}\end{array}$ \\
\hline $\begin{array}{l}\text { Hombres } \\
\text { Alzheimer }\end{array}$ & $\begin{array}{l}\cdots \cdots \\
\cdots \cdots \\
\cdots \cdots \\
\cdots . .\end{array}$ & $\begin{array}{l}\text { / Imit. } \\
\text { / Dep. } \\
\text { / Evit. } \\
\text { / Dep.(r) }\end{array}$ & $\begin{array}{l}3,23^{* *} \\
1,98^{*} \\
1,66^{*} \\
1,39\end{array}$ & $\begin{array}{lll}\ldots . . & \text { / Intr. } \\
\ldots \ldots . & / & \text { Egoís. } \\
\ldots \ldots . & / \ldots . . \\
\ldots . & / & \ldots . .\end{array}$ & $\begin{array}{l}3,26 * * \\
2,27 *\end{array}$ & $\begin{array}{ll}\ldots . . & \text { / Utóp. } 1,70 * \\
\ldots . . & / \ldots \ldots \\
\ldots . & / \ldots \ldots \\
\ldots . & / \ldots \ldots\end{array}$ & $\begin{array}{l}\ldots . . / \text { Pesim. } \\
\ldots \ldots . / \ldots . . \\
\ldots \ldots . / \ldots . . \\
\ldots \ldots . / \ldots .\end{array}$ & 1,18 & 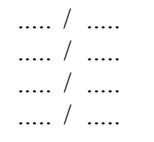 & \\
\hline $\begin{array}{l}\text { Demencia } \\
\text { Mixta-Vasc. }\end{array}$ & $\begin{array}{l}\cdots \cdots \\
\cdots \cdots \\
\cdots \cdots \\
\cdots \cdots\end{array}$ & $\begin{array}{l}\text { / Dep. } \\
\text { / Imit. } \\
\text { / } \ldots \ldots \\
\text { / } \ldots . .\end{array}$ & $\begin{array}{l}1,08 \\
0,93\end{array}$ & $\begin{array}{lll}\ldots . . & \text { / Retr. } \\
\ldots . . / \text { / Egoís. } \\
\ldots . . / \text { Intr. } \\
\ldots . . / \text { Egoc. }\end{array}$ & $\begin{array}{l}2,86^{* *} \\
2,42^{* *} \\
1,88^{*} \\
1,65^{*}\end{array}$ & $\begin{array}{l}\ldots . . \\
\ldots \ldots \\
\ldots .\end{array}$ & $\begin{aligned} \ldots \ldots . & / \text { Pesim. } \\
* 1,75 \text { Enter. } & / \ldots . \\
\ldots . . & / \ldots . \\
\ldots . . & / \ldots .\end{aligned}$ & $2,18^{*}$ & $\begin{array}{ll}\ldots . . & \text { / Rigid. } \\
\ldots \ldots . & \text { Indif. } \\
\ldots . . / & \ldots \ldots \\
\ldots . & \text { / } \ldots . .\end{array}$ & $\begin{array}{l}2,69^{* *} \\
2,03^{*}\end{array}$ \\
\hline
\end{tabular}

Las abreviaturas se corresponden con los elementos enunciados en el punto 11 de la tabla 4.

Sólo se consignan las diferencias significativas: ${ }^{*} \mathrm{p}<0.05 ;{ }^{* *} \mathrm{p}<0.01$; Cursiva: grupo global (TD o TA) con diferencia significativa y grupos internos (MA, HA o DMV) no significativos, aunque del mismo signo y superior a 1,00 . 
$\underline{5.2}$ Factores de personalidad y muerte precoz del padre. En los resultados del contraste entre el subgrupo de enfermos que tienen sólo la muerte del padre antes de los 20 años y la totalidad del grupo control, se observa paradójicamente, en todos los grupos de enfermos $(n=31)$ un menor déficit en el

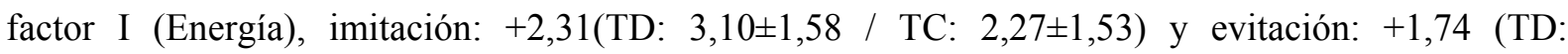
$3,63 \pm 1,95$ / TC: $2,86 \pm 1,90)$.

En el factor V (Apertura Mental), y también para todos los grupos, se constata una generalización de

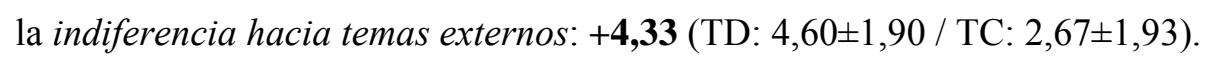

En el factor II (Afabilidad) el déficit continúa centrado los mismos grupos, aunque de forma más

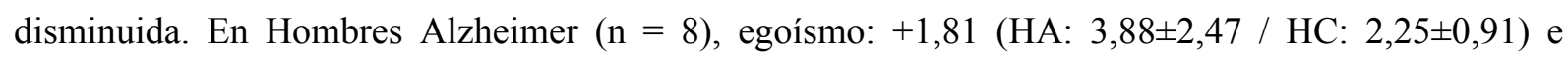

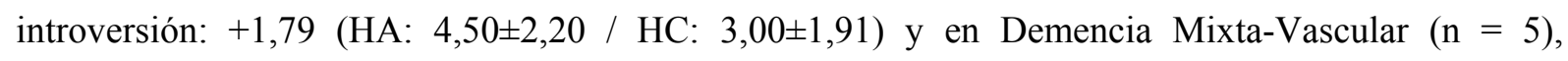

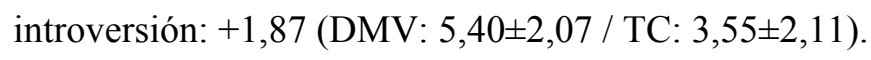

El factor III (Tesón) continúa siendo el de menor déficit; también en Hombres Alzheimer, la irresponsabilidad: $+1,95$ (HA: 3,00 $\pm 2,07$ / HC: $1,55 \pm 0,60$ ).

En el factor IV (Estabilidad emocional) se mantiene en Mujeres Alzheimer ( $\mathrm{n}=18)$ la afectación:

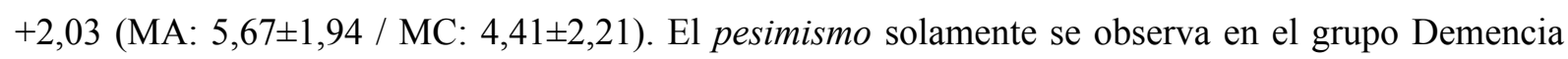

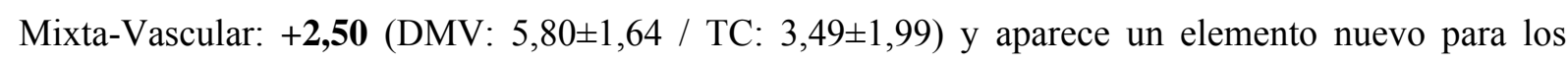

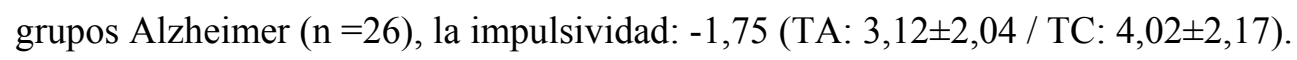

\section{$\underline{5.3 \text { Resultados de los perfiles de personalidad y relación en los ámbitos específicos para el grupo Total }}$}

Alzheimer. Lo más destacable de los ámbitos específicos es: en Matrimonios, la mayor homogeneidad, sin diferencias significativas en los diversos factores, excepto un elemento de dominancia en la

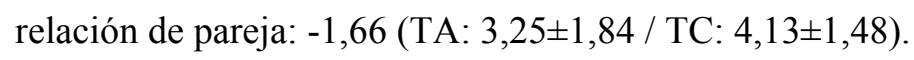


En la Edad de inicio, el mayor déficit en los enfermos $<70$ años del factor I (Energía), imitación:

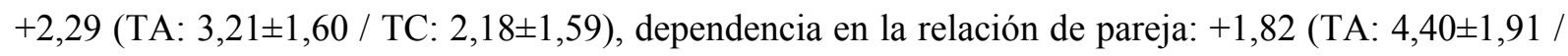

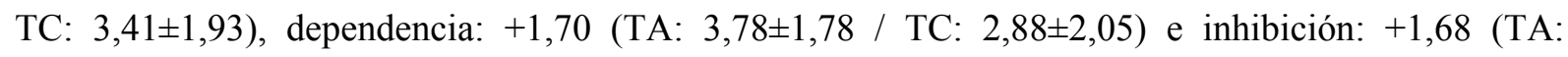

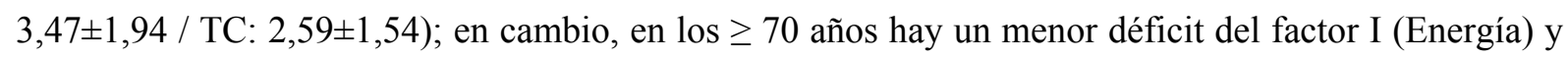

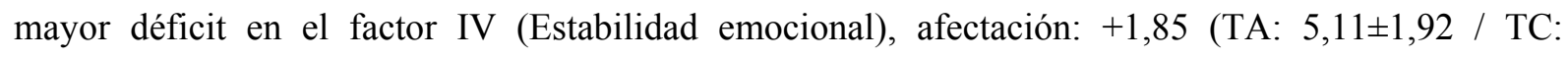

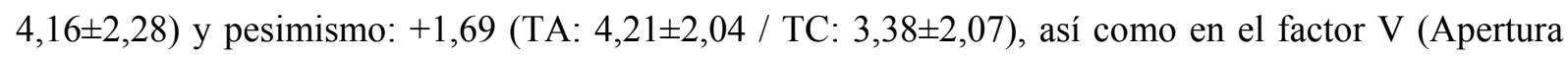

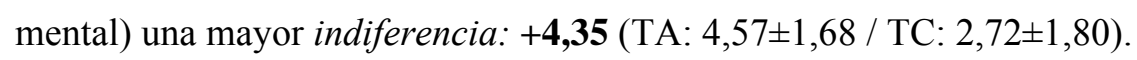

Finalmente, en los enfermos de mayor Nivel educativo resaltar un importante y paradójico déficit,

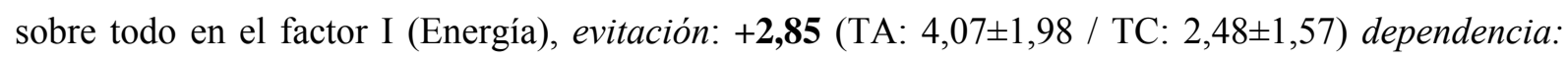

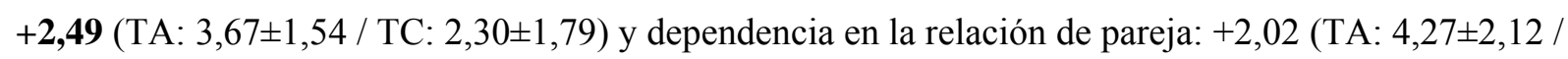
TC: $3,07 \pm 1,66)$.

\section{Discusión}

1) Manifestaciones psíquicas y/o psicosomáticas familiares y personales. Respecto a los antecedentes familiares, los datos significativos sobre trastornos graves (ingresos psiquiátricos, psicosis, suicidios, drogas), así como el mayor número de trastornos psíquicos, para los grupos Alzheimer, apoyarían la tesis de estructuras familiares más frágiles, también en el plano psíquico.

El menor número de trastornos depresivos en el área personal contrasta con los resultados de otras investigaciones ${ }^{33-36}$; sin embargo son coherentes con los antecedentes familiares del estudio, ya que tampoco aparecían como relevantes. Una posible causa de este hecho sea la presencia, en los 
controles, de cuidadores con situaciones estresantes y de personas con déficits leves de memoria, los cuales suelen estar asociados a fenómenos depresivos, aumentando su número en los grupos control. Otra explicación sería el menor número de depresiones, no diagnosticadas, en los grupos de enfermos. Sí que aparecen como más relevantes las respuestas psicosomáticas a los eventos vitales, las cuáles suelen corresponder a una menor capacidad de elaboración psíquica frente a ellos. Este dato podría relacionarse tanto con la mayor fragilidad familiar y personal como con el menor nivel educativo.

2) Familia y estilo educativo. Es notable el déficit en la estructura familiar, en lo referente a la figura paterna, general para todos los grupos, aunque más acusado en Mujeres Alzheimer.

En el análisis de los ámbitos específicos parece clara la relación entre menor nivel educativo y la muerte precoz del padre para todos los grupos. Un caso particular lo constituye el ámbito territorial: las diferencias significativas para Barcelona-ciudad son muy grandes, contrariamente a lo que ocurre en la comarca del Baix Llobregat, donde no se detecta ninguna para ningún grupo. Estos datos podrían tener relación con un factor no controlado en el estudio: la inmigración; la importancia de este factor quedó reflejada en el estudio The Honolulu-Asia Aging Study ${ }^{4}$, al constatarse unas tasas de demencia más altas a las del país de origen, en la población inmigrada, después de 30 años. La muerte precoz del padre y las consecuencias asociadas (dificultades económicas, menor nivel educativo, menor vinculación externa, etc.) podrían favorecer una tasa mayor de demencias, aunque sería preciso un mayor estudio para dilucidar las diferencias entre Barcelona ciudad y Baix Llobregat, ya que no disponemos de datos acerca de qué participantes son nativos o inmigrantes en cada una de las áreas y/o de los grupos de participantes. 
3. Educación, trabajo y relaciones sociales. Los datos, constatando un menor nivel educativo, una menor cualificación profesional y unas peores condiciones de contratación para los grupos grupo Mujeres Alzheimer y Demencia Mixta-Vascular, son coherentes con los estudios citados en la introducción sobre los factores de riesgo ${ }^{7-9}$.

En cuanto a las relaciones y actividades sociales, aspectos que subrayaban los estudios citados anteriormente $^{10-14}$, como elementos protectores de la demencia, los datos no señalan para los grupos Alzheimer diferencias de grado de relaciones sociales (actitud de sociabilidad), aunque si se constata que los grupos Mujeres Alzheimer y Demencia Mixta-Vascular aparecen más centrados en la familia con una menor tasa de participación en entidades.

La hipótesis más plausible, que relaciona unos mayores déficits en educación, status, trabajo, relaciones sociales y demencia, giraría en torno a que todos ellos serían responsables de una menor actividad cerebral, con una menor protección para su conservación ${ }^{37}$. Es preciso considerar no obstante, las menores carencias de todos estos factores sociales para el grupo Hombres Alzheimer.

4. Eventos vitales. De forma global, en el área personal, únicamente Hombres Alzheimer (área laboral) y Demencia Mixta-Vascular (conflictos personales y enfermedades propias) tendrían un mayor número de eventos vitales negativos; en cambio, en el área familiar, habría una mayor problemática familiar para el conjunto de todos los grupos de enfermos con demencia.

Se confirma la hipótesis de los estudios realizados ${ }^{15-16}$ respecto a la presencia de eventos vitales negativos previos al desarrollo de la enfermedad. Los últimos eventos vitales significativos están centrados en el área familiar para los grupos Alzheimer (muerte de familiares y dificultades en la emancipación de los hijos) y en el área personal para Demencia Mixta-Vascular (enfermedad propia). 
Creemos necesario distinguir entre aquellos eventos vitales que tienen un efecto estructural (muerte del padre en la Infancia) y aquellos que ocurren en otras etapas con un efecto más fenomenológico. En los grupos Alzheimer los más recientes (más sentidos) se superponen a los más antiguos (menos sentidos aunque más importantes por las consecuencias). Unos y otros tendrían un efecto sobre el estado de ánimo en forma de depresión, tristeza o simplemente de visión negativa y pesimista.

5. Perfiles de personalidad y relación. Los déficits del factor I (Energía: Imitación, evitación, y dependencia) son bastante consistentes y se repiten en los diferentes ámbitos y subgrupos. Concuerdan con los datos del estudio de la Universidad de Friburgo ${ }^{18}$ que ponían de manifiesto la tendencia a la evitación-sumisión y a depender de otros en decisiones importantes. Respecto al de la Universidad de Viena ${ }^{20}$, que se referían al cónyuge sano como dominante, más bien parece suceder lo contrario: en algunos grupos y ámbitos, es el enfermo el que es visto como dominante en la relación de pareja (es preciso subrayar sin embargo, que el grupo control se puntuó como muy sumiso, lo cual realzó el factor dominancia en los grupos de enfermos). No obstante, este factor de dominancia coexiste con el déficit general del factor I (Energía).

El elemento introversión, que se citaba en el estudio de la Clínica Mayo ${ }^{19}$, está en el presente estudio muy centrado en Hombres Alzheimer y en Demencia Mixta-Vascular, no así en Mujeres Alzheimer. El pesimismo, que también aparecía en el trabajo citado, en los datos presentados sí que es general para todos los grupos de enfermos y estaría en línea con la tesis del estudio de las "Monjas" ${ }^{21}$ : los autores realizaron un estudio con 678 religiosas de entre 75 y 106 años durante 15 años. Algunas de sus conclusiones fueron que las emociones positivas y el optimismo ante la vida son factores que predisponen a la salud mental, se asocian con la longevidad y protegen del Alzheimer. 
En relación a los 4 ámbitos específicos, en la Edad de inicio, los mayores déficits del factor I (Energía) se sitúan en los más precoces, presentando unas características de mayor dependencia; en cambio, los enfermos de inicio tardío presentaban una mayor alteración del factor IV (Estabilidad emocional): afectación y pesimismo; estos datos sugerirían unos factores de riesgo de personalidad diferentes en función de la edad de inicio. En cuanto al Nivel educativo, los mayores déficits del factor I están situados en el nivel superior; esto relativizaría el papel de la educación: la formación de la personalidad aparece como el resultado de diversos factores y no sólo de uno de ellos.

En la muerte precoz del padre, la suposición de que este hecho podría haber afectado a la constitución de su identidad, no parece tener confirmación (el factor I tienen menor intensidad); en los enfermos con muerte precoz del padre, el elemento que se ha visto más reforzado ha sido el de la indiferencia a los temas externos; este segundo aspecto haría referencia al papel del padre como intermediador con el mundo, su ausencia dificultaría los procesos de relación social. Los déficits más elevados del factor I (Energía), II (Afabilidad) y IV (Estabilidad emocional) se encuentran justamente en aquellos enfermos que no han tenido ninguna muerte precoz, ni de padre, ni de madre. La impresión es que se trataría más bien de unos patrones familiares con un estilo educativo de poca vinculación al mundo externo, poco facilitadores de la autonomía y con una visión pesimista, probablemente relacionada con una historia familiar de déficits y carencias diversas. 


\section{Conclusiones}

1) Presencia de factores de riesgo no/biológicos. Se aportan datos suficientes para confirmar su existencia en el ciclo vital de los enfermos con la enfermedad de Alzheimer, buena parte de los cuales han sido documentados también por otras investigaciones.

a) Familiares: El mayor número de muertes precoces parentales, especialmente del padre; el mayor número de antecedentes familiares de trastornos psíquicos graves; la mayor problemática familiar, en cuanto a eventos vitales

d) Sociales: El bajo nivel educativo y ocupación laboral; unas menores relaciones sociales y más centradas en la familia

c) Personales: Los factores de personalidad y relación. I: Dependencia, evitación, imitación; II: Introversión; IV: Pesimismo, afectación; V: Indiferencia hacia temas externos y rigidez

2) Factores que favorecen una mayor predisposición. Los factores de riesgo psicosociales y de personalidad no contradicen los aspectos biológicos de la enfermedad, ya que no se sitúan en el nivel de la etiología. Deben ser contemplados en conjunto como factores de mayor predisposición al desarrollo de una demencia. Los efectos de las carencias en los eventos psicosociales y en la personalidad premórbida de los enfermos de Alzheimer, podrían relacionarse con el paradigma biológico, refiriéndonos a la tesis de la actividad cerebral. Estos factores tendrían en común el propiciar una menor actividad cerebral a lo largo del ciclo vital, lo cual favorecería un mayor deterioro de las capacidades intelectuales.

3) La personalidad premórbida no se corresponde con un trastorno mental específico. Los factores de personalidad premórbida no permiten categorizar un tipo de trastorno psíquico o mental 
específico que estuviera más presente en los enfermos de Alzheimer. Son rasgos comunes, pero que aparecen en ellos de forma más intensa. El déficit de autonomía y de relación no ha generado sintomatología anterior porque, probablemente, han habido elementos con una función de suplencia y compensación: relación de pareja, trabajo, etc. Por otra parte, estos déficits, pueden estar vinculados a la misma estructura familiar, ya sea por las pérdidas parentales ocurridas, por el estilo de relación inter/familiar, o por el grado de vinculación al mundo exterior.

4) Hipótesis de la fragilidad familiar y personal. Los datos presentados aportan la sugerencia de seguir investigando en la hipótesis de la fragilidad familiar y personal, especialmente de lo que ha sido carencial y deficitario, impidiendo que se desarrollase la propia autonomía personal y una mayor y satisfactoria vinculación al mundo externo.

Déficit yoico de autonomía y simbolización por una parte (más presentes en los de inicio precoz) y de retracción social y pesimismo por otra (más presentes en los de inicio tardío), serían los elementos a seguir investigando, conectados con esta fragilidad familiar y personal que se pondría a prueba en la adaptación a los cambios y a las pérdidas que suponen el tránsito de la mediana edad a la Vejez.

5) Posibles intervenciones de prevención. Buena parte de la actividad profesional del autor de la presente investigación ha estado vinculada en los últimos años (1988 / 2002) a las intervenciones de prevención en el campo de las Personas Mayores desde la actividad como psicólogo. Sería deseable encontrar programas operativos que, desde distintos niveles de intervención, pudiesen incidir en la prevención de la enfermedad de Alzheimer. La actual investigación se sitúa en esta línea de trabajo, sugiriendo posibles elementos de prevención: Intervenciones que potencien la autonomía personal, la capacidad de afrontamiento, una mayor estabilidad afectiva y una relación social más intensa. 


\section{Bibliografía}

1. Conde Sala JL. Factores de riesgo y personalidad premórbida en la enfermedad de Alzheimer. Tesis Doctoral, Universidad de Barcelona, 2002. Versión completa en formato digital en Internet: http://tdcat.cesca.es/TDCat-0403102-095159/

2. Conde Sala JL. Familia y Demencia. Intervenciones de apoyo y regulación En: Alberca R, López-Pousa S. editores. Enfermedad de Alzheimer y otras demencias. Madrid: Médica Panamericana, 2002. p. 101-20.

3. Raiha I, Kaprio J, Koskenvuo M, Rajala T, Sourander L. Alzheimer's disease in Finnish twins. Lancet 1996;347:573-8.

4. White L, Petrovitch H, Ross GW, Masaki KH, Abbott RD, Teng EL. et al. Prevalence of dementia in older Japanese-American men in Hawaii: The Honolulu-Asia Aging Study. JAMA 1996;276(12):955-60.

5. Jorm AF. La epidemiología de la enfermedad de Alzheimer y trastornos afines. Barcelona: S.G. Editores; 1994.

6. Conde Sala JL. Personalidad premórbida y factores de riesgo en la enfermedad de Alzheimer. Rev Esp Geriatr Gerontol 1999;34(3):157-61.

7. Evans DA, Hebert LE, Beckett LA, Scherr PA, Albert MS, Chown MJ. et al. Education and other measures of socio-economic status and risk of incident Alzheimer disease in a defined population of older persons. Arch Neurol 1997;54(11):1399-405.

8. Moritz DJ, Petitti DB. Association of education with reported age of onset and severity of Alzheimer's disease at presentation: implications for the use of clinical samples. Am J Epidemiol 1993;137(4):456-62.

9. Ott A, Breteler MM, van Harskamp F, Claus JJ, van der Cammen TJ, Grobbee DE. et al. Prevalence of Alzheimer's disease and vascular dementia: association with education. The Rotterdam study. BMJ 1995;310(6985):970-3.

10. Kondo K, Yamashita I. A case-control study of Alzheimer's Disease in Japan: association with inactive psychosocial behaviors. En: Hasegawa K, Homma A., editors. Psychogeriatrics Biomedical And Social Advances. Amsterdam: Excerpta Medica, 1990. p. 49-53.

11. Bidzan L, Ussorowska D. Risk factors for dementia of the Alzheimer type. Psychiatr Pol 1995;29(3):297306.

12. Shimamura K, Takatsuka N, Inaba R, Iwata H, Yoshida H. Environmental factors possibly associated with onset of senile dementia. Nippon Koshu Eisei Zasshi 1998;45(3):203-12.

13. Helmer C, Damon D, Letenneur L, Fabrigoule C, Barberger-Gateau P, Lafont S, et al. Marital status and risk of Alzheimer's disease: a French population-based cohort study. Neurology 1999;53:1953-8. 
14. Wang H-X, Karp A, Winblad B, Fratiglioni L. Late-life Engagement in Social and Leisure Activities Is Associated with a Decreased Risk of Dementia: A Longitudinal Study from the Kungsholmen Project. Am J Epidemiol 2002;155:1081-7.

15. Shen Y. A case-control study of risk factors on Alzheimer's disease. Multicenter collaborative study in China. Chung Hua Shen Ching Ching Shen Ko Tsa Chih 1992;25(5):284-7.

16. Pecyna SM. Effect of psychological family crises on manifestations of Alzheimer's disease in people of working age. Przegl Epidemiol 1993;47:343-8.

17. Hagnell O, Franck A, Grasbeck A, Ohman R, Ojesjo L, Otterberck L. et al. Senile dementia of the Alzheimer type in the Lundby Study II. An attempt to identify possible risk factors. Eur Arch Psychiatry Clin Neurosci 1992;241(4):231-5.

18. Bauer J, Stadtmuller G, Qualmann J, Bauer H. Premorbid psychological processes in patients with Alzheimer's disease and in patients with vascular dementia. Z Gerontol Geriatr 1995;28(3):179-89.

19. Malinchoc M, Rocca WA, Coligan RC, Offord KF, Kokmen E. Premorbid personality characteristics in Alzheimer's disease: An exploratory case - control study. Behav Neurol 1997;10(4):117-20.

20. Kropiunigg U, Sebek K, Leonhardsberger A, Schemper M, Dal-Bianco P. Psychosocial risk factors for Alzheimer's disease. Psychother Psychosom Med Psychol 1999;49(5):153-9.

21. Danner DD, Snowdon DA. Positive Emotions in Early and Longevity: Findings from the Nun Study. J Pers Soc Psychol 2001;80(5):804-13.

22. Strauss ME, Pasupathi M, Chatterjee A. Concordance between observers in descriptions of personality change in Alzheimer's disease. Psychol Aging 1993;8(4):475-80.

23. Strauss ME, Pasupathi M. Primary caregivers' descriptions of Alzheimer patients' personality traits: temporal stability and sensitivity to change. Alzheimer Dis Assoc Disord 1994;8(3):166-76.

24. Strauss ME, Lee MM, DiFilippo JM. Premorbid personality and behavioral symptoms in Alzheimer disease. Some cautions. Arch Neurol 1997;54(3):257-9.

25. Heun R, Muller H. Interinformant reliability of family history information on psychiatric disorders in relatives. Eur Arch Psychiatry Clin Neurosci 1998;248:104-9.

26. Domènech JM, Granero R. Anàlisi de dades per a la recerca en Psicologia, vol 1 y 2. Esplugues de Llobregat: Signo; 2001.

27. Pardo A, San Martín R. Análisis de datos en Psicología II. Madrid: Pirámide; 1994.

28. Downie NM, Heath RW. Métodos estadísticos aplicados. Madrid: del Castillo; 1971.

29. Pérez Díaz J. Transformaciones sociodemográficas en los recorridos generacionales hacia la madurez. Las generaciones españolas 1906-1945. Tesis Doctoral, Facultad de CC. Políticas y Sociología, UNED; 2001. 
30. Reisberg B, Ferris SH, de León MJ, Crook T. The global deterioration scale for assessment of primary degenerative dementia. Am J Psychiatry 1982;139: 1136-9

31. Caprara GV, Barbaranelli C, Borgogni L, Perugini M. The "Big five Questionnaire". A new questionnaire to assess the five factor model. Pers Individ Dif 1993;15:281-8. Adaptación española de Bermúdez J, BFQ Cuestionario “Big Five”. Madrid: TEA;1995.

32. Costa TP, McCrae RR. The NEO personality inventory manual. Odessa: Psychological Assessment Resources; 1985.

33. Kokmen E, Beard MC, Chandra V, Offord KP, Schoenberg BS, Ballard DJ. Clinical risk factors for Alzheimer's disease: a population based case-control study. Neurology 1991;41(9):1393-7.

34. Van Duijn MC, Clayton DG, Chandra V, Fratiglioni L, Graves AB, Heyman A. et al. Interaction between genetic and environmental risk factors for Alzheimer's disease: a reanalysis of case-control studies. EURODEM Risk Factors Research Group. Genet Epidemiol 1994;11(6):539-51.

35. Speck CE, Kukull WA, Brenner DE, Bowen JD, McCormick WC, Teri L. et al. History of depression as a risk factor for Alzheimer's disease. Epidemiology 1995;6(4):366-9.

36. Tsolaki M, Fountoulakis K, Chantzi E, Kazis A. Risk factors for clinically diagnosed Alzheimer's disease: a case-control study of a Greek population. Int Psychogeriatr 1997;9(3):327-41.

37. Martínez-Lage JM. Educación, reserva cerebral y factores de riesgo de demencia y enfermedad de Alzheimer. Med Clin (Barc) 2001;116:418-21. 